\title{
Concerns about vitamin D and sun exposure behaviour among Australians
}

\section{Tamara Tabbakh, PhD ${ }^{1}$, Melanie Wakefield, AO PhD FASSA ${ }^{2}$, Suzanne J Dobbinson, $\mathbf{P h D}^{3}$}

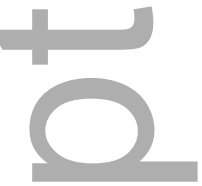

Running head: vitamin D concerns and sun protection

Key words: vitamin D concerns, sun exposure, sunscreen, sun protection, skin cancer prevention

\section{Word count: 3,165 words}

Conflict of interest statement: The authors declare no conflicts of interest.

Ethics approval: Ethical approval to conduct the study was obtained from Cancer Council Victoria's Human Research Ethics Committee (HREC 0018).

Funding: This study was supported by Cancer Council Australia, with additional funding provided by the Australian Department of Health in 2013-14. MW was supported by a National Health and Medical Research Council Fellowship. The funding body was not involved in the analysis and interpretation of data, or in writing the manusscript.

${ }^{1}$ Corresponding author: Centre for Behavioural Research in Cancer, Cancer Council Victoria, Melbourne, Victoria. E-mail: Tamara.Tabbakh@,cancervic.org.au

${ }^{2}$ Centre for Behavioural Research in Cancer, Cancer Council Victoria, Melbourne, Victoria. E-mail: Melanie.Wakefield@cancervic.org.au

${ }^{3}$ Centre for Behavioural Research in Cancer, Cancer Council Victoria, Melbourne, Victoria. E-mail: Suzanne.Dobbinson@cancervic.org.au

This is the author manuscript accepted for publication and has undergone full peer review but has not been through the copyediting, typesetting, pagination and proofreading process, which may lead to differences between this version and the Version of Record. Please cite this article as doi: 10.1002/HPJA.372

This article is protected by copyright. All rights reserved 


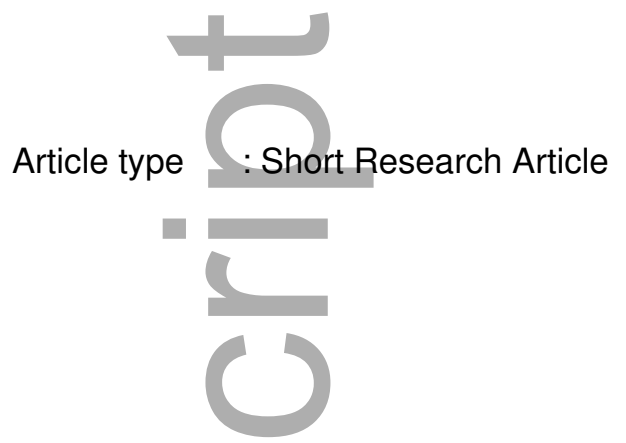

Abstract (243 words)

Issues addressed: Australians' concerns about vitamin D and influence on sun protection were last quantified a decade ago in Queensland amidst media attention on emerging evidence of non-skeletal benefits of vitamin D. Meanwhile these circumstances persist, and impact is worth assessing. Methods: Measures of concern about vitamin D were included in three recent population-based cross-sectional surveys of Australians' sun-related behaviours during summer months (2010-11, 2013-14 and 2016-17). We analysed characteristics, beliefs and behaviours associated with vitamin D concerns regarding regular sunscreen use among adults in 2016-17 (N=3,614). Results: Concerns about vitamin D continued to persist among adults surveyed between 2010-11 and 2016-17. Vitamin D concerns regarding sunscreen use were more common among women, older respondents, and adults with skin that tans or is not susceptible to sunburn. Respondents concerned about vitamin D were more likely to exhibit pro-tanning beliefs, scepticism about sunscreen safety and have attempted a suntan. They were also less likely to use sun protection in everyday activities outdoors and less likely to use sunscreen on summer weekends. Conclusions: Vitamin D concerns related to sunscreen use remained common among Australian adults. Skin cancer control advocates may be reassured that those with greatest skin cancer risk (burn only) were less likely to hold these concerns, while the related infrequent sun protection and tanning among concerned adults is problematic. So what?: Continued education and research is needed to ensure that the general public understand the risks and benefits of sun exposure in Australia.

Keywords: vitamin D, beliefs, sunscreen, sun protection behaviour, skin cancer prevention.

\section{Background}

This article is protected by copyright. All rights reserved 
The main source of vitamin D is skin synthesis following exposure to ultraviolet radiation (UVR), ${ }^{1}$ over-exposure to which is also a risk factor for skin cancer. ${ }^{2}$ Vitamin D has a longestablished important role in musculoskeletal health. ${ }^{3}$ However, the past two decades have witnessed a dramatic rise in epidemiological research on emerging and potential non-skeletal health benefits of vitamin $\mathrm{D},{ }^{4}$ challenging recommended optimal levels of sun exposure. ${ }^{5}$ Findings for a protective role of vitamin D for cardiovascular, respiratory and autoimmune diseases and cancer are mixed, with many relying on observational study designs that limit conclusions about causation. ${ }^{4,6,7}$ Recent findings from the largest randomised controlled trial on vitamin D supplementation conducted in the United States ( $=25,871)$, the VITAL study, showed no reductions in cancer and cardiovascular incidence or mortality among older adults (aged 50 years or above) who took vitamin D supplements relative to those in the placebo group. ${ }^{7}$ However, other studies provide evidence of benefits of vitamin D supplementation for reducing cancer mortality, ${ }^{8}$ all-cause mortality ${ }^{9,10}$ and acute respiratory tract infections. ${ }^{11}$ These recent studies are an improvement on the earlier studies on vitamin D, as they employ more rigorous research designs that are informative about the associations between vitamin $\mathrm{D}$ supplementation and disease. ${ }^{12}$ A plethora of trials on vitamin D supplementation and various health outcomes are expected to be completed in the next decade. ${ }^{4}$ Of particular significance for recommendations in Australia will be the results from the D-Health study of over 20,000 Australians that promises to provide further evidence for the impact of vitamin D supplementation on diagnoses of a host of health conditions, including cancer and heart disease. $^{13}$

The sustained academic interest in vitamin $D$ has been paralleled in the media, with a surge of news stories on the topic. ${ }^{14,15}$ Sentiment analyses of vitamin D articles in popular press suggest a prevalent anti-sun protection theme, with regular dissemination of messages that glorify sun-seeking and encourage Australians to relinquish their sun protection practices in pursuit of vitamin D. ${ }^{16}$ These misleading reports and conflicting messages can propagate confusion about the risks and benefits of sun exposure among the general population ${ }^{17}$ and are concerning for skin cancer prevention advocates in Australia.

Given that dietary sources of vitamin D are limited, achieving vitamin D adequacy via sun exposure while minimising skin cancer risk is challenging. In summer, UVR levels are consistently high across Australia and, theoretically, sun exposure for a few minutes to relatively small areas of the body during mid-morning or mid-afternoon is sufficient for 
adequate vitamin D production among fair-skinned healthy individuals. ${ }^{118}$ However, numerous genetic, metabolic, environmental, behavioural and dietary factors significantly impact cutaneous synthesis of vitamin D. ${ }^{18-20}$ Using the Australian Health Survey data, in 2011-12, approximately one-quarter of Australians were vitamin D deficient ( $<50 \mathrm{nmol} / \mathrm{L})$ : $17 \%$ of participants were described as mildly deficient $(30-49 \mathrm{nmol} / \mathrm{L})$, while $6.5 \%$ were moderately or severely deficient ( $<30 \mathrm{nmol} / \mathrm{L}$ ) based on their serum 25 -hydroxy vitamin $\mathrm{D}$ concentrations. $^{2}$

Further research to provide improved recommendations on optimal UVR exposure specific to individuals' characteristics for vitamin D adequacy and skin cancer risk is limited. ${ }^{18}$ However, findings of a study examining the impact of adhering to the current Australian sun exposure guidelines on serum vitamin D levels found that attaining vitamin D adequacy was easily achievable for most healthy Australians during summer but not winter months. ${ }^{22}$ Therefore, complying with the recommended sun exposure guidelines when the UVR levels are high during summer months in southern states and year-round in lower latitudes is unlikely to be problematic for vitamin D adequacy for the majority of the population.

Nonetheless, cross-sectional studies of Queensland adults surveyed between $2004^{23}$ and $2008^{24}$ suggested that up to half of participants believed that sun protection may result in not getting enough vitamin D. Although different levels of concern might be expected in other states and territories, these findings were concerning given Queensland has one of the highest incidences of skin cancer globally. ${ }^{25}$ In the interim years a prominent discourse on vitamin D and sun exposure has continued in the media as the findings of the latest scientific research on vitamin D and health are revealed. The current study aims to establish if these concerns about vitamin $\mathrm{D}$ have persisted and to what extent sun exposure and sun protection behaviours are associated with these concerns among a large sample of Australian adults.

\section{Methods}

\section{Design and Procedure}

The National Sun Protection Survey (NSPS), an established representative survey conducted triennially since 2003, was used for this paper. Cross-sectional weekly telephone interviews assessing Australians' sun-related attitudes and behaviours were conducted over eight weeks of summer, with a focus on adults surveyed in 2010-11 ( $\mathrm{N}=5,412) ; 2013-14(\mathrm{~N}=5,288)$ and; 2016-17 $(\mathrm{N}=3,614)$ for this analysis. Detailed methods of the NSPS were published 
previously. ${ }^{18}$ Briefly, the sample-frame was initially based on selection of respondents using random digit dialling of landline numbers, with mobile telephones included in 2013-14 onward. A sensitivity analysis of survey findings revealed that inclusion of respondents with a mobile phone is unlikely to affect the assessment of sun-related outcomes over time. Respondents provided details of their sun-related attitudes and sun protection behaviour on the weekend prior to the interview. They were also interviewed about their behaviour more generally during extended and incidental outdoor activities across the summer. Ethical approval to conduct the study was obtained from Cancer Council Victoria's Human Research Ethics Committee (HREC 0018).

Measures

Three measures of vitamin D concerns were included in this analysis. In 2010-11 and 201314 , respondents were asked about their concerns about vitamin D deficiency and reported advice from doctors to get more vitamin D by asking "I am concerned that my current vitamin D levels might be too low" and "Has a doctor ever advised you that you need to get more vitamin D?". In 2016-17, vitamin D concerns regarding regular sunscreen use were assessed by asking respondents if the following statement was true or false - 'People who use sunscreen regularly when they are outdoors do not get enough vitamin D from the sun'. This question served as our primary measure of underlying vitamin D concerns and is thus the focus of this paper.

For our primary measure of vitamin D concerns responses of 'true' or 'can't say' to the statement were classified as 'concerned' given these responses likely reflect a hesitance or aversion to regularly using sunscreen due to concerns about vitamin D; a response of 'false' to the statement was classified as 'not concerned'.

Statistical analysis

Prevalence ratios (PR) were estimated using generalised linear model with log-link (Poisson regression) to describe the characteristics of respondents with vitamin D concerns regarding regular sunscreen use. The relationship between vitamin D concerns regarding regular sunscreen use and sun-related behaviour were also examined using generalised linear models with log-link (Poisson regression). Eight measures of sun-related behaviours were modelled: (i) being unprotected on the most recent day of extended sun exposure (one hour or more); (ii) infrequent (never/rarely/sometimes) use of sun protection during everyday activities for This article is protected by copyright. All rights reserved 
10 minutes or more; (iii) attempted a suntan this season; (iv) made changes to sun protection to get more vitamin D; (v) outdoors on the weekend during peak UVR hours; (vi) amount of time spent outdoors during peak UVR hours on the weekend; (vii) use of sunscreen with at least SPF15+ during outdoor activity on the weekend; and (viii) wore a hat during outdoor activity on the weekend. All models adjusted for age, sex and skin sensitivity as covariates. Skin sensitivity was measured by asking respondents to describe their skin's response after exposure to 30 minutes of strong sunshine during summer and was categorised into three main types: highly sensitive (burn only), moderately sensitive (burn then tan) or not sensitive (tan only). Additionally, behavioural models included the local temperature during peak UVR hours (10am-2pm AEST/11am-3pm DST on the previous Saturday or Sunday for which the sun-related behaviours were reported) obtained from the Bureau of Meteorology. All analyses were performed in IBM SPSS Statistics 24. Generalised linear models with log-link (Poisson regression) were conducted in Stata 14. Results were weighted to be representative of the Australian Estimated Resident Population by age, sex, and geographic location as estimated in June 2010, 2013 and 2016 by the Australian Bureau of Statistics (https://www.abs.gov.au/ausstats).

\section{Results}

The sample comprised adults aged 18-24 years (14\%), 25-44 years (42\%) and 45-69 years $(44 \%)$. Half of participants (50\%) were male and the majority reported skin that was highly $(27 \%)$ or moderately sensitive (44\%). Response rates decreased markedly in the most recent year consistent with broader trends in telephone survey non-response (AAPOR 2010-11: 63\%; 2013-14: 64\%; 2016-17: 11\%). However, in each survey year the sample was broadly representative of the Australian population by socio-economic status and skin type, characteristics not included in the quota-sampling, indicating that sample bias has been minimised(https://www.abs.gov.au/AUSSTATS/abs@.nsf/DetailsPage/2039.0.55.0012006?OpenDo cument).

The proportion of respondents concerned about their vitamin D levels being too low increased from $19 \%$ in $2010-11$ to $28 \%$ in 2013-14. Similarly, there was an increase in respondents reporting their doctor advised them to get more vitamin D during that period $(13 \%$ cf. $25 \%)$.

This article is protected by copyright. All rights reserved 
In 2016-17, 20\% believed the statement 'people who use sunscreen do not get enough Vitamin D from the sun' to be true and 9\% were unsure, totalling $29 \%$ with vitamin D concerns regarding regular sunscreen use. Females, respondents aged 25 years or above and respondents with skin that is moderately or not sensitive to sunburn were more likely to have vitamin D concerns (Table 1).

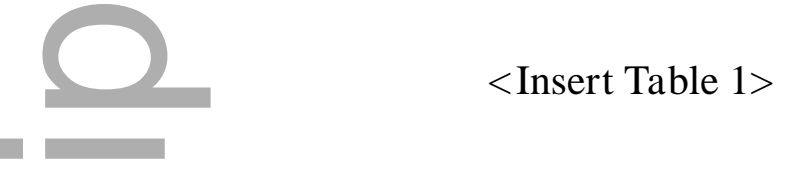

A comprehensive model of tanning, skin cancer and sunscreen beliefs, adjusting for demographic characteristics is included in Table 2. Several determinants of vitamin D concerns emerged from this model. Respondents with vitamin D concerns were more likely to believe that a suntan is healthy (PR: $1.22,95 \%$ CI: 1.08-1.39). Additionally, a negative association between perceived risk of skin cancer and unprotective vitamin D beliefs was observed. When all determinants were considered, perceiving the ingredients in sunscreen to be detrimental for health was the strongest predictor of vitamin D concerns (PR: 2.39, 95\% CI: $2.11-2.72)$.

\section{$<$ Insert Table 2>}

The unadjusted prevalence of skin-cancer related behaviours and the association of vitamin D concerns with these behaviours is summarised in Table 3. Approximately, two-thirds of respondents were outdoors on summer weekends. Less than half of respondents outdoors used SPF15+ sunscreen (35\%) or wore a hat (49\%). Respondents with vitamin D concerns regarding regular sunscreen use were more likely to use sun protection infrequently during their everyday activities (PR: 1.13, 95\% CI: 1.06-1.21), attempt a suntan (PR: 1.36, 95\% CI: 1.09-1.70), make changes to their sun protection to get vitamin D (PR: 2.04, 95\% CI: 1.68 2.47) and were less likely to use sunscreen when outdoors on summer weekends (PR: 0.83, 95\% CI: 0.71-0.98).

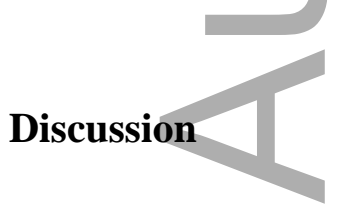

$$
<\text { Insert Table 3> }
$$

This large cross-sectional population-based study of vitamin D concerns found that nearly one in three respondents agreed or were unsure that people who use sunscreen regularly do not get enough vitamin D in 2016-17. Moreover, the observed increase in respondents who 
were concerned that their vitamin D levels were too low between 2010-11 and 2013-14 suggests that these concerns have persisted among Australians for some years. It was notable that adults with vitamin D concerns regarding sunscreen were less likely to use sun protection in several different contexts, namely, on summer weekends during peak UV hours and during incidental activities outdoors. Additionally, these respondents were also more likely to actively seek a tan.

Given our primary measure pertained specifically to concerns about getting enough vitamin D with regular sunscreen use, it was not surprising that adults who held this belief were more likely to agree or be unsure that ingredients in sunscreens are not safe for health. Public mistrust of scientific experts may underlie these sunscreen concerns. ${ }^{26}$ Nonetheless, all ingredients in sunscreen are rigorously tested and approved for safe long-term use ${ }^{27}$ and daily sunscreen use is a proven effective means of reducing UV exposure and risk of developing squamous cell carcinoma and melanoma. ${ }^{28}$ However, concerns specifically about not getting enough vitamin $\mathrm{D}$ are most likely related to the prolonged media attention ${ }^{15}$ and growing evidence of additional health benefits of vitamin $\mathrm{D} .^{8}$

Our findings are broadly consistent with the previous few studies quantifying concerns about getting enough vitamin D while using sun protection among adults in Australia. Although a lower prevalence of these concerns was observed in the current study, the use of a measure with a broader focus on 'sun protection' rather than 'sunscreen use' may partly explain the higher prevalence reported among Queensland adults in $2004^{23}$ and $2008^{24}$. It is unlikely that during the intervening years (2008 to 2010) since the Queensland studies were conducted that there would have been a decrease in concerns about getting enough vitamin D among Australian adults given the continued media attention on this issue. Older adults, and respondents with skin less susceptible to sunburn were more likely to hold concerns about sun protection/sunscreen use and vitamin D in all three studies. Additionally, women were more likely to hold these beliefs in the current study and 2008 Queensland study. ${ }^{24}$ It seems likely the demographic differences in these beliefs can partly be explained by differences in sun protection practices, with those demographic groups more commonly using sun protection ${ }^{29}$ more likely to be concerned about use of sun protection/sunscreen use and not getting enough vitamin D. It was encouraging that respondents with skin that burns easily (i.e. highly sensitive skin) were least likely to have concerns about getting enough vitamin D when using sun protection/sunscreen. ${ }^{23,}{ }^{24}$ Nonetheless, many Australians have a high risk of 
developing skin cancer. ${ }^{30-32}$ Therefore, a higher than negligible risk of skin cancer would be assumed even for respondents reporting their skin tans after exposure to strong sunshine. There was also consistency among these studies; adults with vitamin D concerns regarding sun protection/sunscreen use were more likely to exhibit pro-tanning beliefs and tanning behaviours. Given the long-term education about the harms of UV exposure in Australia, a desire to ignore scientific evidence of the harms of excessive UV exposure- such as the fact that tanning is a sign of DNA damage ${ }^{33}$ in favour of evidence that sun exposure and tanning is healthy ${ }^{34}-$ may underly these pro-tanning beliefs. The current study found concerns about getting enough vitamin D were associated with using less sun protection during numerous contexts adding to the previously identified relationships with intentional sun exposure in pursuit of a tan and reported usual sun protection behaviours. ${ }^{23}, 24$ Interestingly, while the prevalence of concerns about getting enough vitamin D using sun protection among adults in the United States appears to be similar to Australia's, these beliefs have not had an impact on tanning and sun protection behaviours. ${ }^{35}$

Skin cancer control advocates have implemented a number of strategies to keep the community better informed about the harms as well as benefits of sun exposure and vitamin D. Of significance is a collaboration in 2006 with the Australian and New Zealand Bone and Mineral Society, and Osteoporosis Australia to develop a joint position statement on sun exposure and vitamin D risks and benefits. ${ }^{17,36}$ This statement acknowledges the difficulties of providing advice for the population as a whole, whilst recommending use of the Global Solar UV Index (UV Index) to indicate safe levels of sun exposure, namely that sun protection is not required when the UV Index is three or above.

These UV exposure guidelines ${ }^{36}$ provide some tailoring of sun exposure advice to people with specific skin types, but there is limited research on which to base definitive advice on appropriate sun exposure for all skin types and especially for those at greater risk of vitamin D deficiency. ${ }^{37,38}$ There is also limited research to inform the debate as to whether vitamin D supplementation or sun exposure provides the most appropriate benefits for health for populations at risk of low vitamin D. ${ }^{34,39}$ Additionally, the health effects of sun-induced serum vitamin D may differ from vitamin D supplementation, potentially underestimating the benefits of vitamin D on health. Given the impending large trials on vitamin D supplementation and health, ${ }^{4,7,8}$ this discourse is likely to continue and have implications for skin cancer prevention recommendations. Moreover, an update of evidence on the effect of 
sunscreens on vitamin D synthesis is warranted given past sunscreen trials had lower sun protection factors than currently available. ${ }^{27,40} 41-43$ In the meantime, current sun exposure recommendations remain best practice. ${ }^{18,36}$

We note a number of limitations. Firstly, we use a single measure of vitamin D concerns for examining the prevalence, predictors and associated sun protection behaviours among Australian adults. Ideally, future research should examine vitamin D and sun exposure beliefs in a more comprehensive manner, using a validated scale. Additionally, while many researchers treat 'can't say' responses as missing and exclude these from analyses, we included the respondents unable to give a response about the veracity of the statement 'people who use sunscreen regularly when outdoors don't get enough vitamin D from the sun' with those who responded 'true'. We reasoned it was most probable that those who did not wish to respond were most likely to be 'unsure', although some may have not answered for other reasons. However, sensitivity analyses excluding respondents who selected 'can't say', with our three main outcomes revealed similar results to our main findings. Respondents concerned about vitamin D, excluding can't say responses, were statistically significantly more likely to attempt a suntan (PR: 1.55 (1.22-1.95), change their behaviour to get more vitamin D (PR: 2.20 (1.79-2.70) and less likely to use sunscreen when outdoors during peak UVR hours (PR: 0.84 (0.71-0.99). Another key limitation of this study is a reliance on selfreported beliefs and behaviours which although established to be generally valid, may be subject to recall bias. ${ }^{44,45}$ To minimise recall bias the interviews were conducted within three days prior to the reported weekend behaviours. It should also be noted that, although UV was included as a covariate in models of sun-related behaviours the UV levels on summer weekends during peak UV hours (AEST 10am to 2pm/ DST 11am to 3pm) in each state and territory would rarely fall below UV Index $3,{ }^{18}$ and use of sun protection would be appropriately recommended. Finally, there was no direct assessment of respondents' personal or family history of skin cancer. Respondents use of vitamin D supplementation was also not assessed as part of the survey and may also mediate the relationship between concerns about vitamin D and sun protection behaviour.

\section{Conclusions}

This article is protected by copyright. All rights reserved 
This study suggests that concerns about getting enough vitamin D persist among Australian adults. In 2016-17 these beliefs were associated with increased odds of pro-tanning attitudes, scepticism about safety of sunscreen ingredients, suntanning, and infrequent use of sun protection during various outdoor activities, including among those susceptible to the disease. To alleviate these concerns, continued education about the risks and benefits of sun exposure for the general public is needed, along with further research and collaboration between scientific disciplines to determine optimal levels of UV exposure appropriate for all Australians.

\section{Conflict of Interest}

The authors declare no conflicts of interest.

\section{References}

1. Nowson CA, McGrath J, Ebeling PR, et al. Vitamin D and health in adults in Australia and New Zealand: a position statement. Med J Aust. 2012; 196: 686-7.

2. Whiteman DC and Wilson LF. The fractions of cancer attributable to modifiable factors: A global review. Cancer Epidemiology. 2016 44: 203-21.

3. Heaney RP. Vitamin D: How Much Do We Need, and How Much is Too Much? Osteoporos Int. 2000; 11: 553-5.

4. Autier P, Mullie P, Macacu A, et al. Effect of vitamin D supplementation on nonskeletal disorders: a systematic review of meta-analyses and randomised trials. Lancet Diabetes Endocrinol. 2017; 5: 986-1004.

5. Gilchrest BA. Sun exposure and vitamin D sufficiency. American Journal of Clinical Nutrition. 2008; 88: 570S-7S.

6. Theodoratou E, Tzoulaki I, Zgaga L and Ionnidis JP. Vitamin D and multiple health outcomes: umbrella review of systematic reviews and meta-analyses of observational studies and randomised trials. BMJ. 2014; 348.

7. Manson J.E., Cook NR, Lee MI, et al. Vitamin D supplementation and prevention of cancer and cardiovascular disease. The New England Journal of Medicine. 2019; 380: 33-44. 8. Zhang Y, Fang F, Tang J, et al. Association between vitamin D supplementation and mortality: systematic review and meta-analysis. BMJ. 2019; 366: 14673.

This article is protected by copyright. All rights reserved 
9. Bjelakovic G, Gluud LL, Nikolova D, et al. Vitamin D supplementation for prevention of mortality in adults. Cochrane Database Syst Rev. 2014: CD007470.

10. Grant WB and Garland CF. Vitamin D has a greater impact on cancer mortality rates than on cancer incidence rates. BMJ. 2014; 348: g2862.

11. Martineau AR, Jolliffe DA, Hooper RL, et al. Vitamin D supplementation to prevent acute respiratory tract infections: systematic review and meta-analysis of individual participant data. BMJ. 2017; 356: i6583.

12. Booth, C. M., \& Tannock, I. F. (2014). Randomised controlled trials and populationbased observational research: partners in the evolution of medical evidence. British journal of cancer, 110(3), 551-555.13. Neale RE, Armstrong BK, Baxter C, et al. The D-health trial: a randomized trial of vitamin D for prevention of mortality and cancer. Contemp Clin Trials. 2016; 48: 83-90.

14. Scully $M$, Wakefield $M$ and Dixon $H$. Trends in news coverage about skin cancer prevention, 1993-2006: increasingly mixed messages for the public. Aust NZ J Public Health. 2008; 32: 461-6.

15. Scully M, Makin J, Maloney S and Wakefield M. Changes in coverage of sun protection in the news: threats and opportunities from emerging issues. Health Educ Res. 2014; $29: 378-87$.

16. Blake SC, Harding CJ and Doyle Z. A qualitative discourse analysis of safe sun exposure and vitamin D in Australian print media. Australas J Dermatol. 2019.

17. Janda M, Kimlin MG, Whiteman DC, Aitken JF and Neale RE. Sun protection messages, vitamin D and skin cancer: out of the frying pan and into the fire? Med J Aust. 2007; 186: $52-4$.

18. Samanek AJ, Croager EJ, Gies P, et al. Estimates of beneficial and harmful sun exposure times during the year for major Australian population centres. Med J Aust. 2006; 184: $338-41$.

19. Stalgis-Bilinski KL, Boyages J, Salisbury EL, Dunstan CR, Henderson SI and Talbot PL. Burning daylight: balancing vitamin D requirements with sensible sun exposure. Med J Aust. 2011; 194: 345-8.

This article is protected by copyright. All rights reserved 
20. Datta A, Phillipsen PA, Olsen P, et al. Major inter-personal variation in the increase and maximal level of 25-hydroxy vitamin D induced by UVB. Photochem Photobiol Sci. 2016; 15: 536-45.

21. Australian Health Survey: Biomedical Results for Nutrients, 2011-12. Data cubes: Table 1: Nutrient biomarkers by age and then sex, means and medians. Australian Bureau of Statistics.Accessed on: March 6, 2020.

22. Kimlin M, Sun J, Sinclair C, et al. Are the current Australian sun exposure guidelines effective in maintaining adequate levels of 25-hydroxyvitamin D? The Journal of steroid biochemistry and molecular biology. 2015.

23. Janda M, Kimlin M, Whiteman D, Aitken J and Neale R. Sun protection and low levels of vitamin D: are people concerned? Cancer Causes Control. 2007; 18: 1015-9.

24. Youl PH, Janda M and Kimlin M. Vitamin D and sun protection: the impact of mixed public health messages in Australia. Int J Cancer. 2009; 124: 1963-70.

25. Sneyd MJ and Cox B. A comparison of trends in melanoma mortality in New Zealand and Australia: the two countries with the highest melanoma incidence and mortality in the world. BMC Cancer. 2013; 13: 372.

26. Lewandowsky S, Ecker UKH, Seifert CM, Schwarz N and Cook J. Misinformation and Its Correction:Continued Influence and Successful Debiasing. Psychological Science in the Public Interest. 2012; 13: 106-31.

27. Mancebo SE, Hu JY and Wang SQ. Sunscreens: a review of health benefits, regulations, and controversies. Dermatol Clin. 2014; 32: 427-38.

28. Green AC, Williams GM, Logan V and Strutton GM. Reduced melanoma after regular sunscreen use: randomized trial follow-up. J Clin Oncol. 2011; 29: 257-63.

29. Dobbinson S, Wakefield M, Hill D, et al. Prevalence and determinants of Australian adolescents' and adults' weekend sun protection and sunburn, summer 2003-2004. J Am Acad Dermatol. 2008; 59: 602-14.

30. Staples M, Elwood M, Burton R, Williams J, Marks R and Giles G. Non-melanoma skin cancer in Australia: the 2002 national survey and trends since 1985. Med J Aust. 2006; 184: 6-10.

This article is protected by copyright. All rights reserved 
31. Fransen M, Karahalios A, Sharma N, English DR, Giles GG and Sinclair RD. Nonmelanoma skin cancer in Australia. Med J Aust. 2012; 197: 565-8.

32. Whiteman DC, Green AC and Olsen CM. The growing burden of invasive melanoma: Projections of incidence rates and numbers of new cases in six susceptible populations through 2031. J Invest Dermatol. 2016.

33. Friedman B, English JC, 3rd and Ferris LK. Indoor Tanning, Skin Cancer and the Young Female Patient: A Review of the Literature. J Pediatr Adolesc Gynecol. 2015; 28: 275-83.

34. Hoel DG, Berwick M, de Gruijl FR and Holick MF. The risks and benefits of sun exposure 2016. Dermato-endocrinology. 2016; 8: e1248325.

35. Holman DM, Berkowitz Z, Guy GP, Jr., Lunsford NB and Coups EJ. The association between beliefs about vitamin D and skin cancer risk-related behaviors. Prev Med. 2017; 99: 326-31.

36. Australian and New Zealand Bone and Mineral Society, Australasian College of Dermatologists, Cancer Council Australia, Endocrine Society of Australia and Osteoporosis Australia. Position statement - Risks and benefits of sun exposure. Sydney, Australia: Cancer Council Australia, 2016.

37. Xiang F, Lucas R, de Gruijl F and Norval M. A systematic review of the influence of skin pigmentation on changes in the concentrations of vitamin $\mathrm{D}$ and 25-hydroxyvitamin $\mathrm{D}$ in plasma/serum following experimental UV irradiation. Photochem Photobiol Sci. 2015; 14 : 2138-46.

38. Lucas RM, Neale RE, Madronich S and McKenzie RL. Are current guidelines for sun protection optimal for health? Exploring the evidence. Photochem Photobiol Sci. 2018; 17: 1956-63.

39. Khan SR, Whiteman DC, Kimlin MG, et al. Effect of solar ultraviolet radiation exposure on serum 25(OH)D concentration: a pilot randomised controlled trial. Photochem Photobiol Sci. 2018; 17: 570-7.

40. Marks R. Sunscreens and vitamin D levels. J Am Acad Dermatol. 1999; 40: 497.

41. Jayaratne N, Russell A and van der Pols JC. Sun protection and vitamin D status in an Australian subtropical community. Prev Med. 2012; 55: 146-50.

This article is protected by copyright. All rights reserved 
42. Neale RE, Khan SR, Lucas RM, Waterhouse M, Whiteman DC, Olsen CM. The effect of sunscreen on vitamin D: a review. Brit J Dermatol. 2019;181:907-15.

43. Passerson T, Bouillon R, Callender V et al. Sunscreen photoprotection and vitamin D status. Brit J of Dermatol. 2019; 181:916-931.

44. Glanz K, Gies P, O'Riordan DL, et al. Validity of self-reported solar UVR exposure compared with objectively measured UVR exposure. Cancer Epidemiol Biomarkers Prev. 2010; 19: 3005-12.

45. Dobbinson SJ, Jamsen K, Dixon HG, et al. Assessing population-wide behaviour change: concordance of 10-year trends in self-reported and observed sun protection. Int J Public Health. 2013; 59: 157-66.

Table 1. Vitamin D concerns regarding regular sunscreen use among Australian adults, overall and by demographic group

\begin{tabular}{|c|c|c|c|}
\hline \multirow[b]{2}{*}{ Demographic characteristic } & \multicolumn{3}{|c|}{$\begin{array}{l}\text { Vitamin D concerns regarding regular sunscreen } \\
\qquad \text { use }^{\mathrm{a}}\end{array}$} \\
\hline & $\mathrm{N}$ & Unadjusted \% & PR $(95 \% \mathrm{Cl})^{b}$ \\
\hline Overall & 3,614 & 28.7 & \\
\hline \multicolumn{4}{|l|}{ Sex } \\
\hline Male & 1801 & 27.2 & 1.00 (Ref) \\
\hline Female & 1813 & 30.2 & $1.14(1.01-1.28)$ \\
\hline \multicolumn{4}{|l|}{ Age } \\
\hline $18-24$ years & 509 & 18.7 & 1.00 (Ref) \\
\hline $25-44$ years & 1516 & 25.2 & $1.38(1.11-1.72)$ \\
\hline 45-69 years & 1590 & 35.2 & $1.97(1.61-2.41)$ \\
\hline \multicolumn{4}{|l|}{ Skin sensitivityc } \\
\hline Highly sensitive & 983 & 24.6 & 1.00 (Ref) \\
\hline Moderately sensitive & 1597 & 28.6 & $1.16(1.01-1.35)$ \\
\hline Not sensitive & 965 & 31.5 & $1.33(1.14-1.56)$ \\
\hline
\end{tabular}

Note: Bold face font indicates statistical significance at $p<0.05$ level. Unadjusted analysis with no covariates.

a Respondents were classified as concerned if they responded with 'true' or 'can't say' to the item 'people who use

This article is protected by copyright. All rights reserved 
Table 3. Prevalence of sun exposure and sun protection behaviours among adults

\begin{tabular}{|c|c|c|c|}
\hline \multirow{2}{*}{ Dependent variables } & \multicolumn{2}{|c|}{ Unadjusted \% } & \multirow[t]{2}{*}{$\mathrm{PR}(95 \% \mathrm{Cl})^{\mathrm{a}}$} \\
\hline & Concerned $^{b}$ & $\begin{array}{l}\text { Not } \\
\text { concerned }\end{array}$ & \\
\hline \multicolumn{4}{|l|}{ Sun exposure } \\
\hline $\begin{array}{l}\text { Outdoors for extended ( }>1 \text { hour) time and } \\
\text { unprotected }^{c}\end{array}$ & 21.0 & 18.3 & $\begin{array}{c}1.16 \\
(0.99-1.37)\end{array}$ \\
\hline Infrequently protected during everyday activities ${ }^{d}$ & 60.8 & 53.5 & $\begin{array}{c}1.13^{\star \star *} \\
(1.06-1.21)\end{array}$ \\
\hline Tanning and vitamin $\mathrm{D}$ behaviour & & & \\
\hline Attempted a suntan & 13.2 & 10.4 & $\begin{array}{c}1.36^{\star \star} \\
(1.09-1.70)\end{array}$ \\
\hline $\begin{array}{l}\text { Changed sun protection behaviour to get more } \\
\text { Vitamin D }\end{array}$ & 20.7 & 10.0 & $\begin{array}{c}2.04^{\star * *} \\
(1.68-2.47)\end{array}$ \\
\hline \multicolumn{4}{|l|}{ Weekend time outdoors behaviour } \\
\hline Outdoors on the weekend during peak UVR hours & 68.3 & 66.9 & $\begin{array}{c}1.04 \\
(0.98-1.09)\end{array}$ \\
\hline $\begin{array}{l}\text { Mean time spent outdoors }{ }^{\mathrm{e}, \mathrm{f}} \\
\text { (minutes) }\end{array}$ & 110.6 & 107.4 & $\begin{array}{c}B=6.21 \\
(-2.47 \text { to } 14.89)\end{array}$ \\
\hline \multicolumn{4}{|l|}{ Weekend sun protection behaviour } \\
\hline Used sunscreen ${ }^{\dagger}$ (SPF15+) & 35.0 & 44.2 & $\begin{array}{c}0.83^{*} \\
(0.71-0.98)\end{array}$ \\
\hline Wore a hat ${ }^{\dagger}$ & 49.1 & 48.7 & $\begin{array}{c}0.96 \\
(0.82-1.11)\end{array}$ \\
\hline
\end{tabular}

concerned versus not concerned with vitamin D regarding sunscreen use

This article is protected by copyright. All rights reserved 


\section{University Library}

\section{- M M N E R VA A gateway to Melbourne's research publications}

Minerva Access is the Institutional Repository of The University of Melbourne

Author/s:

Tabbakh, T;Wakefield, M;Dobbinson, SJ

Title:

Concerns about vitamin D and sun exposure behaviour among Australians

Date:

2020-07-24

Citation:

Tabbakh, T., Wakefield, M. \& Dobbinson, S. J. (2020). Concerns about vitamin D and sun exposure behaviour among Australians. HEALTH PROMOTION JOURNAL OF AUSTRALIA, 32 (3), pp.399-406. https://doi.org/10.1002/hpja.372.

Persistent Link:

http://hdl.handle.net/11343/276050 